\section{Clinical evaluation of multiparameter correction equations for Goldmann applanation tonometry}

\begin{abstract}
Purpose To evaluate and compare the multiparameter equations in correcting intraocular pressure (IOP) measurements obtained using the Goldmann applanation tonometer (IOPG) for the effects of central corneal thickness (CCT), corneal curvature (R), and age in different ethnic populations.
\end{abstract} Methods Data of IOPG, CCT, R, and age were collected from three clinical centers. The sample size consisted of 945 eyes of 945 glaucoma patients or suspects (669 Europeans, 127 African Americans, and 149 Indians). The 'corrected IOP' was calculated using five multiparameter equations to decrease the association of CCT, R, and age with measured IOP. Regression analyses were performed to calculate variance $\left(r^{2}\right)$ and determine the association of CCT, $R$, and age with IOPG and corrected IOP (residual association).

Results Overall, CCT accounted for the majority of variance in IOPG, while $R$ and age had a much smaller effect, with the combined effect on IOPG ranging from 4.7 to $7.5 \%$ in the three data sets. The residual association of CCT, R, and age with corrected $I O P$ in the three groups ranged from 0.2 to $1.3 \%$ and 0.5 to $1.8 \%$ with the application of the Elsheikh and the Chihara equations, respectively. The residual association of $\mathrm{CCT}$, $R$, and age with corrected IOP calculated using the Ehlers, Orssengo and Pye, and Shimmoyo equations were 7-11.5, 1.8-11.7, and $4.6-8.3 \%$, respectively.

Conclusion The Elsheikh and the Chihara equations better decreased the association of CCT, $R$, and age with measured IOP than the Ehlers, Orssengo and Pye, and Shimmoyo equations.
PG Davey ${ }^{1,2}$, A Elsheikh ${ }^{3,4}$ and DF Garway-Heath ${ }^{4}$

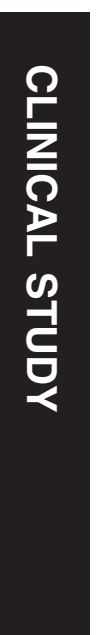

Eye (2013) 27, 621-629; doi:10.1038/eye.2013.23; published online 15 March 2013

Keywords: tonometry; glaucoma; Goldmann applatation tonometer; tonometric correction factors

\section{Introduction}

Intraocular pressure (IOP) measurement is an integral part of the evaluation of patients at risk of glaucoma as IOP is closely related to progressive damage in both primary open-angle and normal tension glaucoma. ${ }^{1-3}$ Current management strategies are based on lowering IOP as the only proven method to slow the progression of glaucomatous damage. ${ }^{4}$ Numerous studies have evaluated the factors that affect the accuracy of IOP measurements made by the clinically available tonometers, and in particular the Goldmann applanation tonometer (GAT), the reference standard in tonometry. ${ }^{5}$ As identified previously, the IOP measurements obtained using the GAT (IOPG) are known to vary with changes in biomechanical parameters that affect corneal stiffness, predominantly the central corneal thickness (CCT), 6,7 central corneal radius of curvature (R), 8,9 hydration, ${ }^{10,11}$ ectasia, ${ }^{12}$ and age. ${ }^{13,14}$ Recently studies have reported that the 'new age' tonometers like the Pascal dynamic contour tonometer (DCT) ${ }^{15}$ and the ocular response analyzer ${ }^{16}$ are less affected by the physiological variations in the biomechanical parameters of the cornea. However, these devices are not available universally and the GAT remains the most commonly used device to measure IOP. ${ }^{5}$
${ }^{1}$ College of Optometry, Western University of Health Sciences, Pomona, CA, USA

${ }^{2}$ Department of Research, Southern College of Optometry, Memphis, TN, USA

${ }^{3}$ School of Engineering, University of Liverpool, Liverpool, UK

${ }^{4} \mathrm{NIHR}$ Biomedical Research Centre for Ophthalmology, Moorfields Eye Hospital NHS Foundation Trust and UCL Institute of Ophthalmology, London, UK

Correspondence: PG Davey, College of Optometry, Western University of Health Sciences, 309 East Second Street, Pomona, CA 91766, USA

Tel: + 19014698473.

E-mail: contact@ pinakin-gunvant.com

Received: 4 July 2012 Accepted in revised form: 22 January 2013 2013 Published online: 15 March 
Studies have previously developed equations to compensate for various corneal biomechanical parameters that influence IOP obtained via applanation tonometry. Some of the correction equations were based on regression analysis of clinical data, ${ }^{17,18}$ results of manometry experiments, ${ }^{19,20}$ mathematical analysis, ${ }^{21}$ or finite element simulations. ${ }^{22}$ Recently Elsheikh and coworkers ${ }^{22}$ proposed a new multiparameter correction equation that mitigates errors induced by $\mathrm{CCT}$, central corneal radius of curvature (R), age, and level of IOP. The equation was expected to diminish the association between IOP measurements made using GAT and CCT, $\mathrm{R}$, and age. The validity of this equation was evaluated in both an in vitro experimental study and a small clinical population. 22,23

The aim of this study was to evaluate the efficacy of the Elsheikh's equation and all other available multiparameter equations developed earlier by Ehlers, ${ }^{19}$ Shimmyo, ${ }^{18}$ Orressengo and Pye, ${ }^{21}$ and Chihara ${ }^{17}$ in various ethnic populations.

\section{Methods}

The data sets used in this study were collected from three different centers: (1) Moorfields Eye Hospital (MEH) NHS Foundation Trust (London, UK); (2) Medical Research Foundation (MRF), Sankara Nethralaya (Chennai, India); and (3) The Eye Center at Southern College of Optometry (Memphis, TN, USA). The data sets obtained from MEH and MRF were obtained for the purposes of other studies. ${ }^{24}$ The MEH data set included White Europeans, whereas the data set from the MRF involved only Indians. To investigate the efficacy of the five correction equations considered in the study in individuals of African-American descent, a chart review was performed at the Eye Center of the Southern College of Optometry to identify patients who met the inclusion criteria of the study. Participants of the three data sets will be referred to as Europeans, Indians, and African Americans, respectively. The study was approved by the Institutional Review Boards and Ethics Committees at the respective study centers and conducted in accordance with the tenets of the Declaration of Helsinki.

Participants included in the study had no history of corneal pathology or surgery and measurements obtained for the study purposes were not made during the postoperative period of intraocular surgeries. The age, the patient's highest recorded untreated IOP (IOPG) and measurements of CCT and R were utilized. The CCT was measured using ultrasound pachmeter at all study centers. The MEH utilized Altair 2000 (Optikon Corp., Kitchener, ON, Canada), MRF utilized BVI Pocket Pachymeter (BV International, Clermont-Ferrand, France), and Southern College of Optometry utilized
DGH-550 Pachette 2 (DGH Technology, Exton, PA, USA). Because $\mathrm{R}$ measurements are not routinely performed in clinical practice, they were not available for the AfricanAmerican data set and a value of $7.8 \mathrm{~mm}$ (the average value for the African-American population as reported by Shimmyo and co-workers ${ }^{18}$ ) was used for calculations.

Only one, randomly selected, eye of an individual was utilized for the study purposes. The sample size consisted of 945 patients, of which 669 were Europeans, 149 Indians, and 127 African Americans. All participants were either diagnosed with, or were at risk of, glaucoma that is ocular hypertension and/or optic disc/visual field features, suggesting glaucoma. Table 1 provides the patient demographics for the three data sets along with the instruments used to measure CCT and R in the three study centers.

\section{Correction equations}

The correction equations that were evaluated in the study included all the multiparameter equations that were available in the literature to correct the IOP as measured using GAT from the errors induced due to variations in corneal biomechanical parameters. Detailed information on the background and development of equations are published elsewhere. ${ }^{17-19,21,22}$ Briefly, the equations provide estimates of the true IOP based on IOP measurements using GAT (IOPG) and corneal biomechanical parameters in the form:

$$
\mathrm{IOPT}=\frac{\mathrm{IOPG}}{A_{\mathrm{CCT}} \cdot A_{\mathrm{R}} \cdot A_{\mathrm{Age}} \cdot A_{\mathrm{IOPG}}}, \text { Reference } 22
$$

$\mathrm{IOPT}=\mathrm{IOPG}+0.071 \times[520-\mathrm{CCT}+0.526 \times(\mathrm{IOPG}-20)]$ $\cdot[0.012 \times(\mathrm{IOPG}-20)+1]$, Reference 19

$\mathrm{IOPT}=\frac{\mathrm{IOPG}+4.15}{\left(\frac{19.09 \times \mathrm{CCT}^{2}}{A(\mu) \times\left(R \times 10^{3}-\mathrm{CTT} / 2\right) \times 10^{4}}+1\right)}$, Reference 17

$$
\begin{aligned}
& \mathrm{IOPT}=\mathrm{IOPG}+ \frac{(550-\mathrm{CCT})}{18 \times \mathrm{e}^{-0.005 \times \mathrm{IOPG}}+0.8 \times(R-7.848837)}, \\
& \text { Reference [18] }
\end{aligned}
$$

$\mathrm{IOPT}=\mathrm{IOPG} \cdot \frac{B}{B_{c}-C_{c}+C}$, Reference 21 
where in equation 1

$$
\begin{aligned}
A_{\mathrm{CCT}}= & 0.68 \times 10^{-6}(\mathrm{CCT}-520)^{2}+1.12 \times 10^{-3}(\mathrm{CCT}-520) \\
& +1.0, A_{R}=1-0.06(R-7.8) \\
A_{\mathrm{Age}}= & 0.3 \times 10^{-6} \mathrm{age}^{3}-88 \times 10^{-6} \mathrm{age}^{2}+0.0085 \mathrm{age} \\
& +0.815, A_{\mathrm{IOPG}}=1.427(\mathrm{IOPG}+3.373)^{-0.119}
\end{aligned}
$$

In equation 3 ,

$$
\begin{aligned}
& \begin{aligned}
A(\mu)= & 0.433047-0.001859 \mu-0.228169 \mu^{2} \\
& +0.237752 \mu^{3}-0.135992 \mu^{4}+0.032129 \mu^{5},
\end{aligned} \\
& \mu=r\left[\frac{12\left(1-v^{2}\right)}{(R-t / 2)^{2} t^{2}}\right]^{1 / 4},
\end{aligned}
$$

and in equation 5

$B=\frac{0.6 \pi R(\mathrm{RCCT} / 2000) \cdot \sqrt{1-v^{2}}}{(\mathrm{CCT} / 1000)^{2}}, C=\frac{\pi R(\mathrm{RCCT} / 2000)^{2} \cdot(1-v)}{\mathrm{A} \cdot \mathrm{CCT} / 1000}$ where $A$ is the area of contact with the tonometer $=\pi \times 1.53^{2}, B_{\mathrm{c}}$ and $C_{C}$ the same as $B$ and $C$, but consider the average CCT and $R$. In all equations, true IOP and IOPG are in $\mathrm{mm} \mathrm{Hg}$, CCT in $\mu \mathrm{m}, R$ in $\mathrm{mm}$, and age in years. Equation 2 was derived in this study based on the correction values presented in the original paper of Ehlers et al. ${ }^{19}$ The equation parameters were derived using the least squares method leading to a root-mean-squared error of $0.12 \mathrm{~mm} \mathrm{Hg}$.

\section{Statistical analysis}

Analysis of variance was utilized to examine the difference in parameters of various groups. Univariate regression analysis was performed to evaluate the effect of three parameters, namely the CCT, R, and age, on both the measured IOP and the corrected values obtained by the five correction equations. However, because CCT, R, and age co-vary, the combined effect of these parameters was analyzed using multiple regression analysis.

Forward stepwise multiple linear regression analysis was performed to evaluate the combined effect of the three parameters on both the measured and corrected IOP. Both the univariate and multivariate analysis results are reported along with the $r^{2}$ values in Table 2 . An $r^{2}$ value of 1.0 is equal to a $100 \%$; therefore, for clarity we will report the $r^{2}$ in percentage values henceforth.

\section{Results}

Table 1 provides mean values of the corneal parameters and the highest recorded IOPG in the three populations considered in the study. Examining for differences in various populations, age was found to be significantly different between centers (ANOVA $F=4.46 ; P=0.011$ ). Tukey contrasts revealed that mean age was significantly greater in the African-American group when compared with Indians, whereas the difference between the Europeans and Indians was not significant. The difference in radius of curvature was not significant among Europeans and Indians (ANOVA F = 2.89; $P=0.09$ ). The CCT and IOP were significantly different between all the groups (ANOVA F $=64.80 ; P<0.0001$ and $\mathrm{F}=165.08 ; P<0.0001$, respectively).

\section{Effect of corneal parameters and age on measured IOP}

Table 2 provides the $r^{2}$, which indicates the amount of variance in measured IOP accounted for by the individual parameters using both univariate and multivariate regression analyses. Evaluating the results of univariate regression analysis, it is found that CCT accounts for the most variance in measured IOP in all three populations compared with the effect of $\mathrm{R}$ and age. Figure 1 shows the scatter plots of association of IOP with CCT, R, and age for all the ethnic populations evaluated. Evaluating the scatter plots, it is found that the associations with CCT and R were in the same direction in all three populations.

As noted by several earlier studies, $, 3,6,7$ there was a significant positive association between CCT and IOPG, with the IOPG on average being greater in individuals with thicker CCT when compared with those with thinner CCT. The CCT accounted for 7.2, 3.6, and $6.6 \%$ of the IOP variance in Europeans, Indians, and African Americans, respectively.

The association between $\mathrm{R}$ and IOPG was also consistent with earlier findings, $, 18,25,26$ with IOP being greater in steeper than average corneas and lower in flatter corneas. However, this trend was not statistically significant with R accounting for only $0.3 \%$ of the variance in IOP in Indians and $0.0007 \%$ in Europeans.

Similarly, the association of IOPG with age was not statistically significant for all populations. Evaluating the

Table 1 Mean ( \pm standard deviation) of parameters utilized in the study

\begin{tabular}{lrrr}
\hline & $\begin{array}{c}\text { European } \\
(\mathrm{n}=669)\end{array}$ & $\begin{array}{c}\text { African } \\
\text { Americans } \\
(\mathrm{n}=127)\end{array}$ & $\begin{array}{c}\text { Indians } \\
(\mathrm{n}=149)\end{array}$ \\
\hline $\begin{array}{l}\text { Age in years } \\
\begin{array}{l}\text { Central corneal thickness } \\
\text { in microns }\end{array}\end{array}$ & $559( \pm 15)$ & $61( \pm 17)$ & $55( \pm 11)$ \\
$\begin{array}{l}\text { Corneal radius of } \\
\text { curvature in mm }\end{array}$ & $7.7( \pm 0.3)$ & $\begin{array}{c}\text { Not } \\
\text { available }\end{array}$ & $7.7( \pm 0.3)$ \\
$\begin{array}{l}\text { Highest recorded } \\
\text { intraocular pressure } \\
\text { measured using GAT }\end{array}$ & $17.6( \pm 4.5)$ & $19.2( \pm 5.3)$ & $25.4( \pm 5.4)$ \\
in mm Hg & & & \\
\hline
\end{tabular}


Table 2 Association of central corneal thickness, curvature and age with corrected and uncorrected intraocular pressure in various populations

\begin{tabular}{|c|c|c|c|c|c|c|}
\hline \multirow{3}{*}{ Dataset } & \multicolumn{6}{|c|}{$r$ - square of linear regression analysis (\%) } \\
\hline & \multicolumn{3}{|c|}{ Univariate analysis } & \multicolumn{3}{|c|}{ Multivariate analysis } \\
\hline & CCT & $R$ & Age & $C C T+R$ & $C C T+A g e$ & $C C T+R+A g e$ \\
\hline \multicolumn{7}{|l|}{ Europeans $\mathrm{n}=669$ individuals } \\
\hline Uncorrected Goldmann IOP & $\underline{7.2}$ & $<0.1$ & 0.5 & $\underline{6.9}$ & $\underline{7.5}$ & $\underline{7.5}$ \\
\hline \multicolumn{7}{|l|}{ Corrected IOP } \\
\hline Elsheikh equation & 1.1 & 0.2 & 0.1 & 1.2 & 1.2 & 1.3 \\
\hline Chihara's equation & 1.5 & 0.1 & 0.4 & 1.6 & 1.9 & 1.98 \\
\hline Ehlers equation & $\underline{11.2}$ & 0.2 & 0.1 & $\underline{11.3}$ & $\underline{11.5}$ & $\underline{11.5}$ \\
\hline Orrssengo and Pye equation & $\overline{2.0}$ & 0.0 & 0.2 & $\underline{2.1}$ & $\underline{\underline{2.3}}$ & $\overline{2.4}$ \\
\hline Shimmyo's equation & $\underline{\overline{7.5}}$ & 0.2 & 0.1 & $\overline{7.6}$ & $\overline{7.8}$ & $\underline{7.9}$ \\
\hline \multicolumn{7}{|l|}{ Indians $\mathrm{n}=149$ individuals } \\
\hline Uncorrected Goldmann IOP & 3.6 & 0.3 & $<0.1$ & 4.6 & 3.8 & 4.7 \\
\hline \multicolumn{7}{|l|}{ Corrected IOP } \\
\hline Elsheikh equation & 0.1 & $<0.1$ & 0.2 & 0.1 & 0.2 & 0.3 \\
\hline Chihara's equation & 0.1 & 0.2 & 0.1 & 0.4 & 0.3 & 0.6 \\
\hline Ehlers equation & $\underline{10.8}$ & 2.5 & 0.6 & $\underline{11.6}$ & $\underline{10.9}$ & $\underline{11.8}$ \\
\hline Orrssengo and Pye equation & $\underline{9.3}$ & 1.1 & 0.6 & $\underline{9.9}$ & $\underline{9.4}$ & 9.6 \\
\hline Shimmyo's equation & $\overline{7.2}$ & 2.3 & 0.5 & $\overline{8.2}$ & $\overline{7.3}$ & 8.3 \\
\hline \multicolumn{7}{|l|}{ African Americans $\mathrm{n}=127$ individuals } \\
\hline Uncorrected Goldmann IOP & 6.6 & Not available & 0.5 & Not available & 6.6 & Not available \\
\hline \multicolumn{7}{|l|}{ Corrected IOP } \\
\hline Elsheikh equation & 1.7 & 1.9 & 3.1 & & & \\
\hline Chihara's Equation & 1.6 & 0.2 & 1.7 & & & \\
\hline Ehlers equation & 7.0 & 0.1 & 7.1 & & & \\
\hline Orrssengo and Pye equation & 1.8 & $<0.1$ & 1.9 & & & \\
\hline Shimmyo's equation & 4.5 & $<0.1$ & 4.6 & & & \\
\hline
\end{tabular}

CCT is central corneal thickness in microns; $R$ is mean corneal radius of curvature; age is in years. The $r$-square of linear regression analysis is given for both univariate and multivariate association of various parameters and IOP represents the amount of variance accounted for. An $r$-square value of 1.0 is equal to a 100 percent of variance accounted. The statistical significance is evaluated using the regression analysis $P$-value. The $r$-square value in bold represents a $P$ value of $<0.05$, numbers is bold and italics represents a $P$ value of $<0.01$. The numbers in bold, italics and underlined or bold, italics and double underlined represents $P$-value of $<0.001$ or $<0.0001$ respectively.

scatter plots, it is found that the association of IOPG and age was not in the same direction for all populations. Both Europeans and Indians showed a positive association for IOPG with age $\left(r^{2}=0.005\right.$ and 0.00006), whereas in African Americans the measured IOPG had a negative slope, indicating a decrease in IOPG with increasing age, albeit the association was not statistically significant (see Figure 2 and Table 2).

\section{Multivariate analysis}

Table 2 presents the change in $\mathrm{r}^{2}$ with the addition of parameters in the multiple regression analysis. As CCT accounted for the greatest amount of variance in IOP when compared with age and $\mathrm{R}$, it was decided to start the multivariate linear regression analysis model with CCT and add either age or R or both in assessing variance in measured IOP. In Europeans, the association between age and IOPG is greater than $\mathrm{R}$ and IOPG in univariate analysis (0.5 vs $0.0007 \%$ ). Adding age to CCT in multivariate linear regression analysis led to an increase in accounting of IOP variance of $0.3 \%$ (from 7.2 to $7.5 \%$ ). Adding corneal radius of curvature to the multivariate model that included CCT and age did not account for any further variance.

In contrast, $\mathrm{R}$ accounted for greater variance than age in the univariate linear regression analysis in Indians, and when added to the multivariate regression model of CCT and IOPG, it accounted for an additional $1 \%$ in variance leading to a total of $4.6 \%$. Adding age to the multivariate model of CCT and corneal radius of curvature resulted in a total variance of $4.7 \%$ in IOPG.

In the African-American population, adding age to the univariate model of CCT and IOP did not account for any additional variance. The effect of $\mathrm{R}$ could not be evaluated in this population as it was not available. 


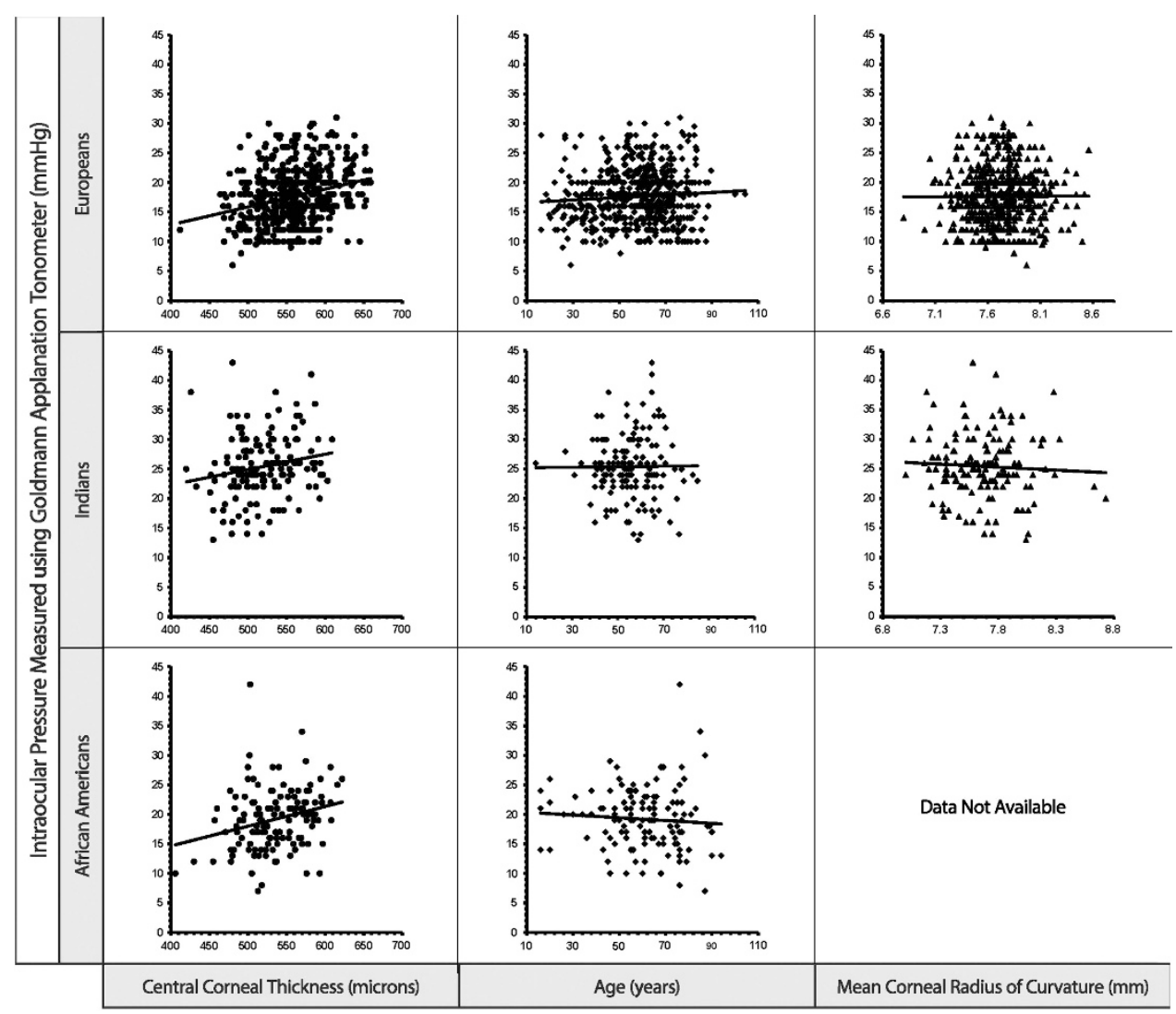

Figure 1 Effect of central corneal thickness, age and mean corneal radius of curvature on intraocular pressure measured using the Goldmann applanation tonometer in different ethnic populations. Results are shown for 669 Europeans, 149 Indians and 127 African Americans.

\section{Residual effect of corneal parameters and age on corrected IOP}

Table 2 further provides the $r^{2}$ of univariate and multivariate analysis of CCT, R, and age on corrected IOP as obtained by the five correction equations. Figure 2 shows the scatter plots of the residual effect of CCT on corrected IOP as obtained using various corrections in Europeans, Indians, and African Americans.

\section{Univariate analysis}

Evaluating the residual effect of CCT on corrected IOP in scatter plots, it is found that Elsheikh equation had a flat line or a weak negative slope indicating optimal correction or slight overcorrection in different populations and the Chihara equation had a flat line or weak positive slope indicating optimal or slight under correction of IOP. Whereas the slope was negative when examining corrected IOP obtained using the Ehlers, Orressengo and Pye, and Shimmyo equations indicating overcorrection of IOP. The residual association of CCT and corrected IOP in various populations obtained using the Elsheikh equation and Chihara equation ranged from 0.1 to $1.7 \%$ and from 0.1 to $1.6 \%$, respectively. The residual associations between the CCT and corrected IOP of Ehlers, Orressengo and Pye, and Shimmyo equations were in the ranges 7.0-11.2, 1.8-9.3, and 4.5-7.5\% respectively. On the other hand, $\mathrm{R}$ and age did not have any significant residual associations with corrected IOP as obtained by any of the five correction equations considered.

\section{Multivariate analysis}

As noted previously, in the univariate analysis, both $\mathrm{R}$ and age only accounted for a small residual variance in corrected IOP. The addition of these parameters to CCT in the multivariate analysis accounted for only small increments in the r-square values. The r-square values obtained using multivariate regression analysis indicate that the residual associations between corrected IOP and the parameters of $\mathrm{CCT}$, age and $\mathrm{R}$ were in the ranges $0.2-3 \%$ (Elsheikh equation) and 0.6-1.9\% (Chihara 


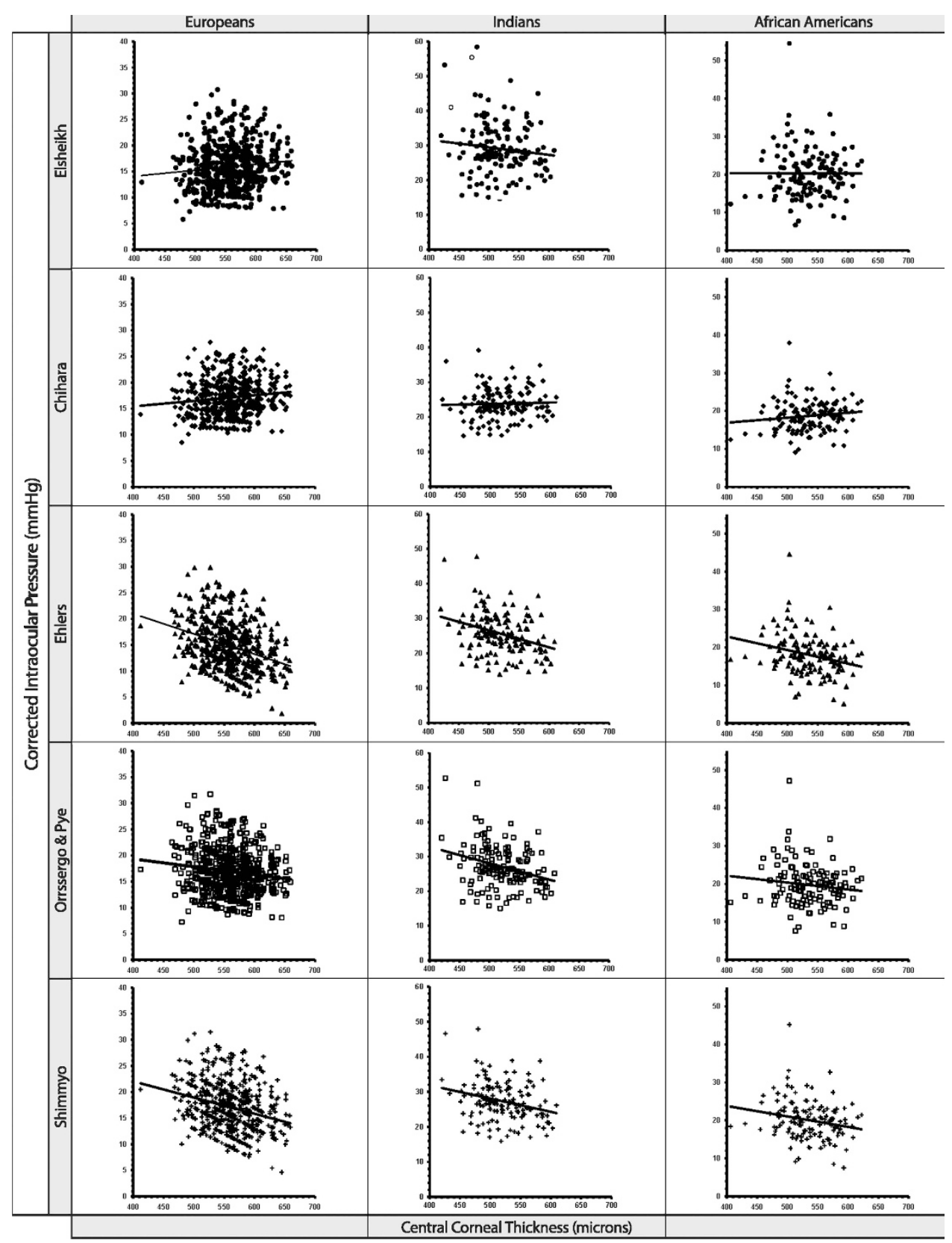

Figure 2 The residual effect of central corneal thickness on corrected intraocular pressure calculated using various multiparameter equations. Results are shown for 669 Europeans, 149 Indians and 127 African Americans.

equation). The Orssengo and Pye equation had residual associations in the range $1.8-9.5 \%$ with the association of various parameters with corrected IOP being weaker in European and African American populations when compared to the Indian population. On the other hand, while using the Ehlers and the Shimmyo equations, the combined association of CCT, R and age was greater for corrected IOP when compared to uncorrected IOP with r-square ranging from 7.1 to $11.5 \%$ and 4.6 to $8.3 \%$, respectively, indicating an overcorrection.

\section{Discussion}

Reduction of IOP is an important part of glaucoma management but the accuracy of its measurements is affected by a number of error sources including the variations in biomechanical parameters such as corneal thickness, curvature and age. 15,27 Whilst methods that improve the estimate of IOP and decrease the effects of errors are a welcome development, it should be remembered that the error reduction is an average effect 
across a population; corrections at an individual level result in measurements that are likely to be more accurate, however, the corrections are made with parameters associated with biomechanical properties, rather than the biomechanical properties themselves, so correction errors at the individual level may occur. This study evaluated by both univariate and multivariate analyses the association of biomechanical parameters with IOP as measured using the GAT (IOPG) and after correction using the five available multi-parameter correction equations. ${ }^{17-19,21,22}$ The univariate analysis has the advantage of examining the associations individually and determining the trends of association with the examined parameter, whereas multiple regression analysis evaluates the combined effect of different parameters. Both univariate and multivariate analysis indicate that the CCT is the major source of error in IOP with $\mathrm{R}$ and age contributing to a lesser extent.

Examining the results of the univariate analysis we find there was a significant positive association between CCT and measured IOP in all populations, whereas there was a negative linear association between $\mathrm{R}$ and measured IOP which was not statistically significant. The level of association between measured IOP and corneal parameters is reported by numerous other publications and the results of the present study are within the range of association reported. ${ }^{13,19,25}$

The association of age with IOP is more recently researched and some studies have shown age-related biomechanical changes in cornea ${ }^{23,28}$ and a weak, albeit significant, positive association between age and measured IOP. ${ }^{13,14}$ In this study, the level of association is below what was reported previously and is not consistent across the three ethnic groups considered. While there is a weak positive association between age and measured IOP in both Europeans and Indians, the association has a negative slope in African Americans and, contrary to earlier reports, the association between age and the IOG is not statistically significant in this study. A number of reasons could explain this outcome. The present study was not designed to examine the association between age and measured IOP in various ethnicities and the mean age was significantly different in African Americans compared to Europeans and Indians. Furthermore, the issue of age-related biomechanical changes to the cornea is complex with parameters such as the long-term exposure to high IOP in glaucoma and the level of glucose in the aqueous possibly having an effect. ${ }^{29,30}$ This study included both glaucoma patients and suspects and did not exclude diabetics and that may have had an effect on the outcome. It is indeed possible that age-related changes are different in different ethnic groups based on the differences in nutritional, environmental or genetic issues, but this hypothesis requires further study.
Examining the multiple regression equations we find that although CCT is the major source of error, adding $\mathrm{R}$ and/or age accounts for additional variance in measured IOP both in Europeans and Indians. This makes a case for the need of multi-parameter equations that accounts for age and $\mathrm{R}$ along with CCT. The association of $\mathrm{R}$ and IOPG in African Americans could not be evaluated as the measurements of $\mathrm{R}$ were not available. This shortfall could be addressed in a future study especially because the mean $\mathrm{R}$ is flatter in African American group when compared to Europeans. ${ }^{18}$

The multi-parameter correction factors for tonometry can simultaneously account for errors induced by various parameters thus improving the accuracy of measurements obtained with GAT; a device that is considered the reference standard in clinics worldwide. ${ }^{5}$ The use of multi-parameter correction equations on the IOPG could decrease the level of association with CCT, age and $\mathrm{R}$ that influence the IOP measurement. This study evaluated the use of five multi-parameter equations in a large clinical population with different ethnic groups. Overall, the Elsheikh and Chihara equations were able to decrease the association of IOPG with CCT, $\mathrm{R}$ and age. These results are consistent with our prior publication that evaluated in vitro the efficacy of the same correction equations. ${ }^{23}$

The application of the Ehlers and Shimmoyo correction equations to IOPG increased the association between corrected IOP and the parameters examined (CCT, R and age) in all three populations. While the values of r-square with uncorrected IOP were 7.5, 4.7 and $6.6 \%$ for the European, Indian and African American groups, respectively, the r-square increased to $11.5,11.7$ and $7.1 \%$ with Ehlers equation, and 7.9, 8.3 and $4.6 \%$ with Shimmyo equation. Overall, the residual error was greater when using the Ehlers correction than the Shimmyo equation (about 3$4 \%$ ). Further, the Orssengo and Pye equation overcorrected IOP for the effect of CCT. This can be seen in univariate analysis as the association between CCT and the IOP corrected with Orssengo and Pye equation had a negative slope similar to that obtained with Ehlers and Shimmyo corrections albeit with smaller value (see Figure 2).

Both the multivariate and univariate analyses indicated that all correction equations varied in their degree of efficacy when applied in different ethnic populations. This may be due to sample size differences between populations, or 'true' ethnic differences in corneal dimensions and biomechanical properties in the populations examined. Nevertheless since there were consistent decreases in the strength of association between CCT, R and age when IOP corrected using the Elsheikh and Chihara equations, these equations are expected to provide a closer approximation of 'true' IOP than GAT measurements. 
This study has the advantages of examining in a large population the efficacy of various correction factors. The data were obtained by various practitioners and using different equipment in different ethnic populations, thus simulating a true clinical picture. One of the limitations of the study is the retrospective design, which made it not possible to obtain $\mathrm{R}$ in African Americans. However, the small effect of this parameter on the association with IOP indicated clearly that this omission in the data was unlikely to considerably affect the final study outcomes.

Both the univariate and multivariate analyses showed that CCT was a more important source of error in tonometry than R and age. However, there are other error sources that influence the values obtained by GAT and cannot yet be accounted for including the measurement error, calibration issues, misalignment of the tonometric mires, valsalva maneuver, nervousness and forced eyelid closure. Furthermore, the correction equations are still unable to address the effects on IOPG due to a number of factors that influence corneal stiffness such as the long exposure to high levels of IOP in glaucomatous eyes, the chronic use of topical glaucoma medications, variations in corneal asphericity, wound healing following surgery or injury, ${ }^{31,32}$ diurnal hydration, ${ }^{10,11}$ ectasia $^{12,33}$ and diabetes. ${ }^{29,30}$ Research is needed to assess the significance of these effects and to quantify them if necessary before their effect on IOPG could be considered.

The accurate measurement of IOP is an important component in the management of all forms of glaucoma. While it has been claimed that new contact tonometers, in particular the DCT can provide IOP estimates that are less affected than GAT by the effects of corneal stiffness, ${ }^{34,35}$ GAT still maintains its worldwide position as the reference standard in tonometry ${ }^{5}$ and the most commonly used device in clinical practice. The results of this study, which identify the suitability of two multiparameter correction equations in reducing the stiffnessrelated errors in IOP measurement, should therefore be considered a step in the right direction to potentially lead to better clinical management of glaucoma. Additionally one has to remember that the CCT values are an independent predictor of development of primary open angle glaucoma. ${ }^{36}$

\section{Summary}

\section{What was known before}

- Goldmann applanation tonometry is influenced by corneal thickness, radius of curvature and age. Numerous correction factors are available.

\section{What this study adds}

- Clinical evaluation of existing correction factors and provides evidence for which factors perform better.

\section{Acknowledgements}

The authors wish to thank Dr Aachal Kotecha for sharing the data from Moorfields Eye Hospital.

\section{References}

1 Hong S, Seong GJ, Hong YJ. Long-term intraocular pressure fluctuation and progressive visual field deterioration in patients with glaucoma and low intraocular pressures after a triple procedure. Arch Ophthalmol 2007; 125(8): 1010-1013.

2 Caprioli J, Coleman AL. Intraocular pressure fluctuation a risk factor for visual field progression at low intraocular pressures in the advanced glaucoma intervention study. Ophthalmology 2008; 115(7): 1123-1129.

3 Leske MC, Heijl A, Hyman L, Bengtsson B, Dong L, Yang Z. EMGT Group. Predictors of long-term progression in the early manifest glaucoma trial. Ophthalmology 2007; 114(11): 1965-1972.

4 Leske MC, Heijl A, Hussein M, Bengtsson B, Hyman L, Komaroff E. Early Manifest Glaucoma Trial Group. Factors for glaucoma progression and the effect of treatment: the early manifest glaucoma trial. Arch Ophthalmol 2003; 121(1): 48-56.

5 ISO. Ophthalmic instruments-tonometers. Vol. ISO8612(E). Copyright Office: Switzerland 2001.

6 Whitacre MM, Stein RA, Hassanein K. The effect of corneal thickness on applanation tonometry. Am J Ophthalmol 1993; 115(5): 592-596.

7 Ko YC, Liu CJ, Hsu WM. Varying effects of corneal thickness on intraocular pressure measurements with different tonometers. Eye (Lond) 2005; 19(3): 327-332.

8 Mark HH. Corneal curvature in applanation tonometry. Am J Ophthalmol 1973; 76: 223-224.

9 Kaufmann C, Bachmann LM, Thiel MA. Comparison of dynamic contour tonometry with Goldmann applanation tonometry. Invest Ophthalmol Vis Sci 2004; 45(9): 3118.

10 Hamilton KE, Pye DC, Aggarwala S, Evian S, Khosla J, Perera R. Diurnal variation of central corneal thickness and Goldmann applanation tonometry estimates of intraocular pressure. J Glaucoma 2007; 16(1): 29-35.

11 Kotecha A, Crabb DP, Spratt A, Garway-Heath DF. The relationship between diurnal variations in intraocular pressure measurements and central corneal thickness and corneal hysteresis. Invest Ophthalmol Vis Sci 2009; 50(9): 4229-4236.

12 Nash IS, Greene PR, Foster CS. Comparison of mechanical properties of keratoconus and normal corneas. Exp Eye Res 1982; 35(5): 413-424.

13 Kotecha A, White ET, Shewry JM, Garway-Heath DF. The relative effects of corneal thickness and age on Goldmann applanation tonometry and dynamic contour tonometry. Br J Ophthalmol 2005; 89(12): 1572-1575.

14 Tonnu PA, Ho T, Newson T, El Sheikh A, Sharma K, White E et al. The influence of central corneal thickness and age on intraocular pressure measured by pneumotonometry, noncontact tonometry, the Tono-Pen XL, and Goldmann applanation tonometry. Br J Ophthalmol 2005; 89(7): 851-854.

15 Boehm AG, Weber A, Pillunat LE, Koch R, Spoerl E. Dynamic contour tonometry in comparison to intracameral IOP measurements. Invest Ophthalmol Vis Sci 2008; 49(6): 2472-2477. 
16 Medeiros FA, Weinreb RN. Evaluation of the influence of corneal biomechanical properties on intraocular pressure measurements using the Ocular Response Analyzer. J Glaucoma 2006; 15: 364-370.

17 Chihara E. Assessment of true intraocular pressure: the gap between theory and practical data. Surv Ophthalmol 2008; 53(3): 203-218.

18 Shimmyo M, Ross AJ, Moy A, Mostafavi R. Intraocular pressure, Goldmann applanation tension, corneal thickness, and corneal curvature in Caucasians, Asians, Hispanics, and African Americans. Am J Ophthalmol 2003; 136(4): 603-613.

19 Ehlers N, Bramsen T, Sperling S. Applanation tonometry and central corneal thickness. Acta Ophthalmol (Copenh) 1975; 53(1): 34-43.

20 Foster PJ, Wong JS, Wong E, Chen FG, Machin D, Chew PT. Accuracy of clinical estimates of intraocular pressure in Chinese eyes. Ophthalmology 2000; 107(10): 1816-1821.

21 Orssengo GJ, Pye DC. Determination of the true intraocular pressure and modulus of elasticity of the human cornea in vivo. Bull Math Biol 1999; 61(3): 551-572.

22 Elsheikh A, Alhasso D, Gunvant P, Garway-Heath D. Multiparameter correction equation for Goldmann applanation tonometry. Optometry Vis Sci 2011; 88(1): E102-E112.

23 Elsheikh A, Gunvant P, Jones SW, Pye D, Garway-Heath DF. In-vitro experimental assessment of multi-parameter correction factors for Goldmann tonometry. Journal of Glaucoma 2013; 22(2): 156-163.

24 Gunvant P, O'Leary DJ, Baskaran M, Broadway DC, Watkins RJ, Vijaya L. Evaluation of tonometric correction factors. J Glaucoma 2005; 14: 337-343.

25 Gunvant P, Baskaran M, Vijaya L, Joseph IS, Watkins RJ, Nallapothula $\mathrm{M}$ et al. Effect of corneal parameters on measurements using the pulsatile ocular blood flow tonograph and Goldmann applanation tonometer. $\mathrm{Br} \mathrm{J}$ Ophthalmol 2004; 88(4): 518-522.
26 Liu J, Roberts CJ. Influence of corneal biomechanical properties on intraocular pressure measurement: quantitative analysis. J Cataract Refract Surg 2005; 31(1): 146-155.

27 Holden BA, Mertz GW, McNally JJ. Corneal swelling response to contact lenses worn under extended wear conditions. IOVS 1983; 24: 218-226.

28 Elsheikh A, Alhasso D, Rama P. Biomechanical properties of human and porcine corneas. Exp Eye Res 2008; 86(5): 783-790.

29 Krueger RR, Ramos-Esteban JC. How might corneal elasticity help us understand diabetes and intraocular pressure? J Refract Surg 2007; 23(1): 85-88

30 Goldich Y, Barkana Y, Gerber Y, Rasko A, Morad Y, Harstein $\mathrm{M}$ et al. Effect of diabetes mellitus on biomechanical parameters of the cornea. J Cataract Refract Surg 2009; 35(4): 715-719.

31 Dupps Jr WJ, Wilson SE. Biomechanics and wound healing in the cornea. Exp Eye Res 2006; 83(4): 709-720.

32 Bryant MR, Szerenyi K, Schmotzer H, McDonnell PJ Corneal tensile strength in fully healed radial keratotomy wounds. Invest Ophthalmol Vis Sci 1994; 35(7): 3022-3031.

33 Andreassen TT, Hjorth Simonsen A, Oxlund H. Biomechanical properties of keratoconus and normal corneas. Exp Eye Res 1980; 31(4): 435-441.

34 Luce DA. Determining in vivo biomechanical properties of the cornea with an ocular response analyzer. J Cataract Refract Surg 2005; 31(1): 156-162.

35 Punjabi OS, Kniestedt C, Stamper RL, Lin SC. Dynamic contour tonometry: principle and use. Clin Exp Ophthalmol 2006; 34(9): 837-840.

36 Gordon MO, Beiser JA, Brandt JD, Heuer DK Higginbotham EJ, Johnson CA et al. The Ocular Hypertension Treatment Study: baseline factors that predict the onset of primary open-angle glaucoma. Arch Ophthalmol 2002; 120(6): 714-720. 